Proceedings of the International School and Conference on Optics and Optical Materials, ISCOM07, Belgrade, Serbia, September 3-7, 2007

\title{
Propagation of Spatial Solitons with Nonlinear Kerr Effect in Thermal Medium
}

\begin{abstract}
A. Gharaati, P. Elahi* and S. Cari
Department of Physics, Payame Noor University, Shiraz, Iran

In this paper, the propagation and behavior of spatial solitons of inhomogeneous nonlinear Schrödinger equation in thermal medium will be investigated analytically. The theoretical model is established by considering the change of refractive index of medium due to heat deposition of entrance beam. The soliton like solution in such thermal medium was derived. The effect of input pump power and beam radius on the behavior of solitons will be studied finally.
\end{abstract}

PACS numbers: 42.65.Sf, 42.65.Tg, 42.65.Wi

\section{Introduction}

The study of electromagnetic waves in nonlinear self-focusing Kerr media has been one of the considerable recent interests. One of the widely studied in nonlinear phenomena is the propagation of spatial solitons. A light beam can form a dielectric waveguide for itself in which the refractive index is greater at the center of the beam than at its wings. Since the light beam in this self-formed dielectric waveguide can propagate without spreading the phenomenon of "self-trapping" of an optical beam was thereby predicted to occur [1]. One may also understand this phenomenon as a dynamic balancing of two opposing tendencies, namely, the tendency for the beam to expand due to dispersion, and the tendency for the beam to contract due to self-focusing; see e.g. [2]. Spatial solitons are not only investigated theoretically but they have also been observed experimentally [3-6]. There are many applications for spatial solitons like pulse compression $[7,8]$, optical switching [9], logic devices [10], etc.

The heat deposition in the propagating medium due to input beam can produce some thermo-optic effects like thermal lensing. Thermal lensing converts the homogeneous refractive index of medium to inhomogeneous one [11, 12].

${ }^{*}$ corresponding author; e-mail: pelahi@spnu.ac.ir 
In this paper firstly, the thermal change of refractive index due to thermal effects for a slab crystal has been obtained. The spatial nonlinear Schrödinger equation in such inhomogeneous medium then has been derived. After solving that equation, the effect of input pump power and pump radius on the behavior of propagation of spatial solitons has been investigated.

\section{Thermal lensing model}

The steady state heat conducting equation in the medium with thermal conductivity $k$ is [13]:

$$
k \nabla^{2} T(x, y, z)=-Q(x, y, z),
$$

where $Q(x, y, z)$ is heat power density due to input beam thermal load and $T(x, y, z)$ is temperature distribution. We consider the slab crystal with dimensions $2 b \times c \times L$. The intensity is homogeneous along the $Y$-direction and in a length of $2 a<2 b$ along the $X$-direction in Fig. 1 .

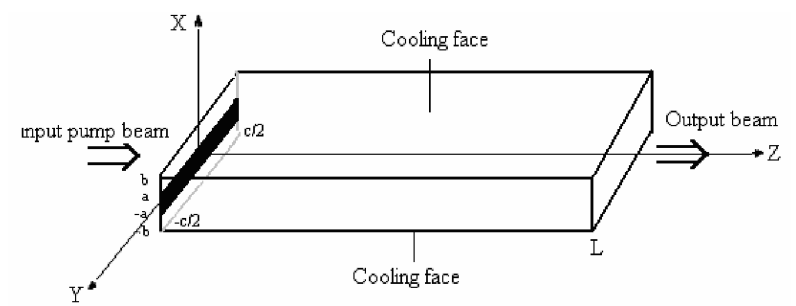

Fig. 1. Schematic diagram of end-pumped slab. The pump region area is $2 a \times c$.

By neglecting the pump divergence along the direction of beam propagation, the total heat load can be written as $Q=\eta P / 2 a c L$ in the pump region and $Q=0$ in the non-pump region where $\eta$ is heat generation coefficient. By considering the two large coolants on the two faces and the direction of heat flow in the $X$ direction, the solution of Eq. (1) can be written as $T_{\text {pump }}=-\frac{Q}{2 k} x^{2}+A x+B$ and $T_{\text {nonpump }}=C x+D$, where $A, B, C$, and $D$ are constants which can be calculated by the following boundary conditions which are the continuity of the temperature and the first derivative on the boundary mean $\left.T_{\text {pump }}\right|_{r=a}=\left.T_{\text {nonpump }}\right|_{r=a}$ and $\left.\frac{\mathrm{d} T_{\text {pump }}}{\mathrm{d} x}\right|_{r=a}=\left.\frac{\mathrm{d} T_{\text {nonpump }}}{\mathrm{d} x}\right|_{r=a}$, respectively, and Newtonians boundary conditions, $\left.k \frac{\mathrm{d} T_{\text {nonpump }}}{\mathrm{d} x}\right|_{r=b}=h\left(T_{\mathrm{c}}-\left.T_{\text {nonpump }}\right|_{r=b}\right.$, where $T_{\mathrm{c}}$ is the ambient temperature and $h$ is the convective heat transfer coefficient. After applying the above boundary condition the temperature distribution in the pump region and outside will be

$$
\begin{aligned}
& T_{\text {pump }}=-\frac{Q}{2 k} x^{2}+Q a\left(\frac{1}{h}+\frac{b}{k}-\frac{a}{2 k}\right)+T_{\mathrm{c}}, \\
& T_{\text {nonpump }}=-\frac{Q a}{k} x+Q a\left(\frac{1}{h}+\frac{b}{k}\right)+T,
\end{aligned}
$$


respectively. The temperature distribution can change the refractive index of medium. The temperature depending change of refractive index can be written as $[13]$ :

$$
n(x)-n_{0}=\left[T_{\text {pump }}(x)-T_{0}\right] \frac{\mathrm{d} n}{\mathrm{~d} T},
$$

where $T_{0}$ is temperature at $x=0$ and $\frac{\mathrm{d} n}{\mathrm{~d} T}$ is a thermo-optic characteristic of the medium. So by inserting Eq. (2) for temperature distribution in the pump region into Eq. (3) we find

$$
n(x)-n_{0}=\left[-\frac{Q}{2 k} x^{2}-Q a\left(\frac{1}{h}+\frac{b}{k}-\frac{a}{2 k}\right)+T_{\mathrm{c}}\right] \frac{\mathrm{d} n}{\mathrm{~d} T} .
$$

By definition $\alpha=-\frac{Q}{k} \frac{\mathrm{d} n}{\mathrm{~d} T}$ and $\beta=-\left[Q a\left(\frac{1}{h}+\frac{b}{k}-\frac{a}{2 k}\right)-T_{\mathrm{c}}\right] \frac{\mathrm{d} n}{\mathrm{~d} T}$, Eq. (4) can be rewritten as

$$
\Delta n(x)=n_{0}-n(x)=\frac{1}{2} \alpha x^{2}+\beta .
$$

So due to heat load, the refractive index is inhomogeneous and behaves like a lens-like medium.

\section{Spatial nonlinear Schrödinger equation in inhomogeneous medium}

The steady state wave equation in a centrosymmetric nonlinear medium with considering $\chi^{(3)}$ as higher order of susceptibility is

$$
\nabla^{2} E+\frac{\omega^{2}}{C^{2}}\left(n_{0}^{2}+\chi^{(3)}|E|^{2}\right) E=0
$$

where $n_{0}$ is refractive index of medium and $c$ is the speed of light. We consider the polarized electric field solution of Eq. (6) as

$$
E=\frac{1}{2}\left\{A(x, z) \exp \left[\mathrm{i}\left(k_{0} z-\omega t\right)\right]+\text { c.c. }\right\},
$$

where $A(x, z)$ is the envelope amplitude of wave and $k_{0}=\frac{n_{0} \omega}{c}$ is the wave number. By defining

$$
X=k_{0} x, \quad Z=k_{0} z, \quad q(X, Z)=\sqrt{\frac{n_{2}}{n_{0}}} A(x, z) \quad \text { and } \quad n_{2}=\frac{\chi^{(3)}}{2 n_{0}},
$$

and then substituting Eq. (7) into Eq. (6) and using Eq. (8) we find

$$
\mathrm{i} \frac{\partial q}{\partial Z}+\frac{1}{2} \frac{\partial^{2} q}{\partial X^{2}}+|q|^{2} q=0
$$

This dimensionless equation is called the spatial nonlinear Schrödinger equation and the uniform moving solution can be considered as

$$
q(X, Z)=\eta \operatorname{sech}\left(\eta\left(X+v Z-X_{0}\right)\right) \exp \left(-\mathrm{i}\left(v X+\frac{1}{2}\left(v^{2}-\eta^{2}\right) Z\right)\right),
$$

where $v$ is the velocity of the soliton, $\eta$ is amplitude and $X_{0}$ is constant. Now we suppose the refractive index of medium is inhomogeneous and consider the inhomogeneous refractive index as $n_{0}+\Delta n(X)$, in which $n(X) \ll n_{0}$. By neglecting $\Delta n(X)^{2}$, and by using the steady state wave equation and Eqs. (7) and (8), we 
find

$$
\mathrm{i} \frac{\partial q}{\partial Z}+\frac{1}{2} \frac{\partial^{2} q}{\partial X^{2}}+|q|^{2} q=V(X) q
$$

where $V(X)=-\Delta n(X) / n_{0} k^{2}$. In analogy with the homogeneous Schrödinger equation, the spatial solution can be assumed to have the following form:

$$
q(X, Z)=\eta \operatorname{sech}(\eta(X-\bar{X}(Z))) \exp (\mathrm{i} G(Z) X+H(Z)),
$$

where $\bar{X}(Z)$ is the average location of soliton in $X$ direction. By inserting the above equation in Eq. (11), after separating the real and imaginary parts, we obtain two equations in the following forms:

$$
\begin{aligned}
& \frac{\mathrm{d} \bar{X}(Z)}{\mathrm{d} Z}=G(Z), \\
& X \frac{\mathrm{d} G(Z)}{\mathrm{d} Z}+\frac{\mathrm{d} H(Z)}{\mathrm{d} Z}+\frac{1}{2} G^{2}(Z)+V(X)-\frac{1}{2} \eta^{2}=0 .
\end{aligned}
$$

By derivation of Eq. (13) with respect to $Z$ and Eq. (14) with respect to $X$ we find the dynamic behavior of average location of solitons as

$$
\frac{\mathrm{d}^{2} \bar{X}(Z)}{\mathrm{d} Z}=-\frac{\mathrm{d} V(\bar{X})}{\mathrm{d} \bar{X}}
$$

The above equation is Newtonian's like second law.

According to Eq. (5), and by introducing $\bar{\alpha}=\alpha / n_{0} k^{4}$, and $\bar{\beta}=\beta / n_{0} k^{2}$, the potential in average location of soliton will be

$$
V(\bar{X})=-\frac{1}{2} \bar{\alpha} \bar{X}^{2}+\bar{\beta} .
$$

So by using Eqs. (15) and (16) we find

$$
\begin{aligned}
\bar{X}(Z) & =A_{1} \sin \sqrt{\bar{\alpha}} Z+A_{2} \cos \sqrt{\bar{\alpha}} Z, \\
G(Z) & =A_{1} \sqrt{\bar{\alpha}} \cos \sqrt{\bar{\alpha}} Z-A_{2} \sqrt{\bar{\alpha}} \sin \sqrt{\bar{\alpha}} Z, \\
H(Z) & =\frac{-3}{8} \sqrt{\bar{\alpha}} \sin (2 \sqrt{\bar{\alpha}} Z)\left(A_{2}^{2}-A_{1}^{2}\right)-\frac{3}{\alpha} \sqrt{\bar{\alpha}} A_{1} A_{2} \cos (2 \sqrt{\bar{\alpha}} Z) \\
+ & \frac{1}{4} Z \bar{\alpha}\left(A_{2}^{2}+A_{1}^{2}\right)+\frac{1}{2} Z\left(1-\bar{\alpha} X^{2}\right)+A_{3},
\end{aligned}
$$

where $A_{1}, A_{2}$, and $A_{3}$ are constant values defined with initial conditions.

\section{Results}

Based on the theoretical results, we used a typical crystal slab with dimensions $2 b=10 \mathrm{~mm}, c=20 \mathrm{~mm}$ and $L=100 \mathrm{~mm}$. The thermal conductivity is $0.13 \mathrm{~W} /(\mathrm{cm} \mathrm{K})$ and the coolant temperature has been considered as $T_{\mathrm{c}}=300 \mathrm{~K}$. Figures 2a,b show the beam intensity for a $10 \mathrm{~W}$ input beam with $a=0.5 \mathrm{~mm}$ (a) and $a=1 \mathrm{~mm}$ (b). As shown in these figures, for such input pump regime, the pump radius does not influence the behavior of the solitons in the medium.

Figures 3a,b show the beam intensity in the medium for a $50 \mathrm{~W}$ input beam with (a) $a=0.5 \mathrm{~mm}$ and (b) $a=1 \mathrm{~mm}$. As shown the effect of the pump radius in the behavior of soliton is more pronounced by increasing the input pump power. The decreasing beam radius gives the increase in the variation of the beam along 


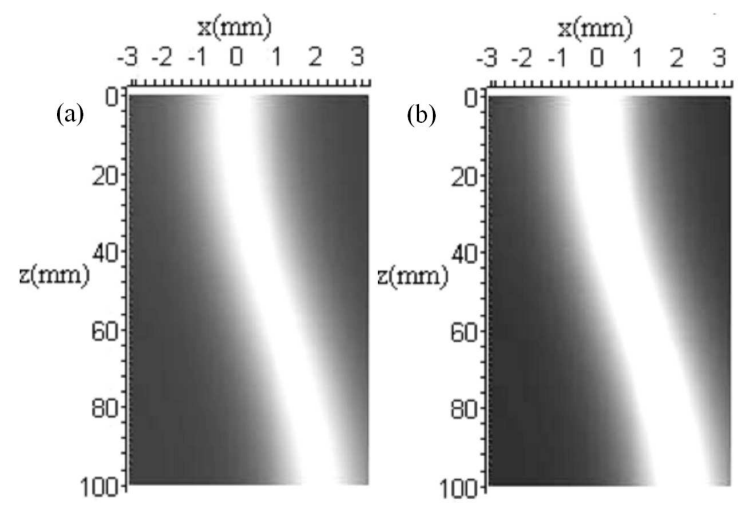

Fig. 2. Beam intensity for $10 \mathrm{~W}$ input power with (a) $a=0.5 \mathrm{~mm}$ and (b) $a=1 \mathrm{~mm}$.
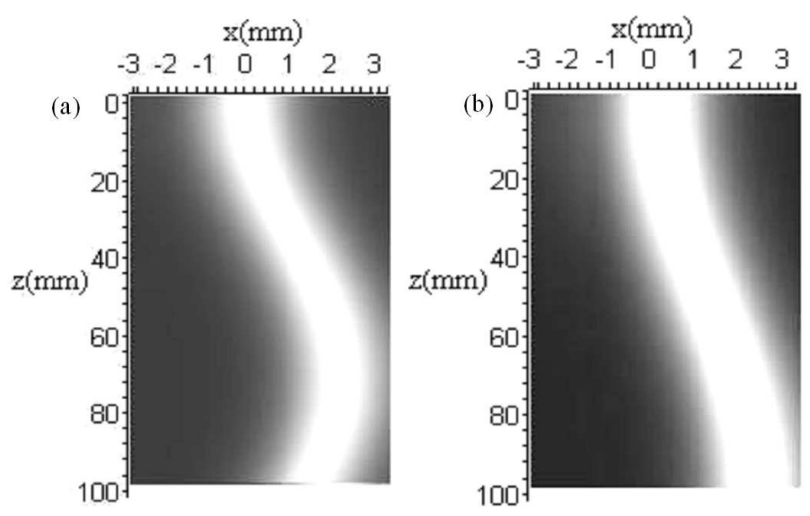

Fig. 3. Beam intensity for $50 \mathrm{~W}$ input power with (a) $a=0.5 \mathrm{~mm}$ and (b) $a=1 \mathrm{~mm}$.

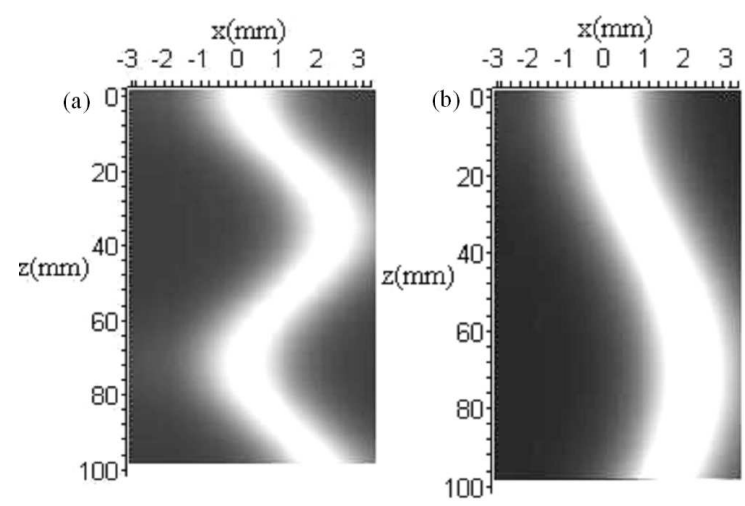

Fig. 4. Beam intensity for $100 \mathrm{~W}$ input power with (a) $a=0.5 \mathrm{~mm}$ and (b) $a=1 \mathrm{~mm}$.

the $X$ axis in the medium for such input power. Figures $4 \mathrm{a}$,b show the beam intensity of the soliton for $100 \mathrm{~W}$ and (a) $0.5 \mathrm{~mm}$ beam radius and (b) $1 \mathrm{~mm}$. 
By comparing Figs. 2a, 3a, and 4a it is obvious that the variation of soliton along $X$-axis will be more pronounced in high-power regime.

\section{Conclusions}

In this paper, we investigated the spatial soliton of nonlinear Schrödinger equation in the inhomogeneous refractive index medium. The influence of input power and radius on the behavior of spatial solitons is studied analytically. We showed that the influence of input beam radius on the behavior of soliton is more noticeable in high-power regime with respect to low-power one. For wider beam the effect of input power is smaller and can be neglected.

\section{References}

[1] R.Y. Chiao, E. Garmire, C.H. Townes, Phys. Rev. Lett. 13, 479 (1964).

[2] R.Y. Chiao, in: Spatial Soliton, Eds. S. Trillo, W. Torruellas, Springer-Verlag, Berlin 2001, p. 1.

[3] J.S. Aitchison, A.M. Weiner, Y. Silberberg, M.K. Oliver, J.L. Jackel, D.E. Leaird, E.M. Vogel, P.W. Smith, Opt. Lett. 15, 471 (1990).

[4] J.S. Aitchison, Y. Silberberg, A.M. Weiner, D.E. Leaird, M.K. Olivier, J.L. Jackel, P.W.E. Smith, J. Opt. Soc. Am. B 8, 1290 (1991).

[5] A. Barthelemy, S. Maneuf, C. Froehly, Opt. Commun. 55, 201 (1985).

[6] R. De La Fuente, A. Barthelemy, C. Froehly, Opt. Lett. 16, 793 (1991).

[7] M.M. Cerimele, M.L. Chiofalo, F. Pistella, S. Succi, M.P. Tosi, Phys. Rev. E 62, 1382 (2000).

[8] D.H. Reitze, A.M. Weiner, D.E. Leaird, Opt. Lett. 16, 1409 (1991).

[9] J. Scheuer, M. Orenstein, Opt. Lett. 24, 1735 (1999).

[10] G. Cancellieri, F. Chiaraluce, E. Gambi, P. Pierleoni, J. Opt. Soc. Am. B 12, 1300 (1995).

[11] W.K. Koechner, D.K. Rice, Appl. Opt. 9, 2548 (1970).

[12] H. Nadgaran, P. Elahi, PRAMANA-J. Phys. 66, 513 (2006).

[13] W. Koechner, Solid-State Laser Engineering, 5th ed., Springer-Verlag, Berlin 1999. 